$51 \mid 2013$

Grammaire et enseignement du français langue étrangère et seconde. Permanences et ruptures du XVle au milieu du XXe siècle (I)

\title{
L'héritage du modèle latin dans les grammaires françaises à la Renaissance
}

\section{Bernard Colombat}

\section{(2) OpenEdition \\ Journals}

\section{Édition électronique}

URL : https://journals.openedition.org/dhfles/3710

DOI : $10.4000 /$ dhfles.3710

ISSN : 2221-4038

\section{Éditeur}

Société Internationale pour l'Histoire du Français Langue Étrangère ou Seconde

Édition imprimée

Date de publication : 1 février 2013

Pagination : 11-38

ISSN : 0992-7654

Référence électronique

Bernard Colombat, "L'héritage du modèle latin dans les grammaires françaises à la Renaissance », Documents pour l'histoire du français langue étrangère ou seconde [En ligne], 51 | 2013, mis en ligne le 31 janvier 2017, consulté le 21 septembre 2021. URL : http://journals.openedition.org/dhfles/3710 ; DOI : https://doi.org/10.4000/dhfles.3710

Ce document a été généré automatiquement le 21 septembre 2021.

(c) SIHFLES 


\title{
L'héritage du modèle latin dans les grammaires françaises à la Renaissance
}

\author{
Bernard Colombat
}

\section{Introduction}

1 La grammaire française a été créée par un transfert à partir de la grammaire latine, par ce qu'on peut appeler un «transfert de modèle ». Auroux (1994) utilise le concept de "grammaire latine étendue", "latine» parce que le cadre est latin, comme le reconnaissent beaucoup d'auteurs, par ex. R. Estienne décrivant le contenu de sa grammaire inspirée principalement de Meigret et de Sylvius :

Le tout auons mis par ordre, et traicte a la maniere des Grammaires Latines, le plus

clerement et facilement qu'auons peu. (R. Estienne, 1557, au lecteur : 3-4)

2 «étendue " parce que le modèle est appliqué à d'autres langues, le français n'étant qu'une parmi d'autres.

\subsection{Quel héritage?}

On sait depuis longtemps que les deux sources principales sont deux artes: l'Ars Donati et l'Ars Prisciani, la première d'entre elles étant subdivisée en deux parties : l'Ars minor, traité élémentaire sous forme de questions réponses centré sur les parties du discours; l'Ars maior, traité plus étendu sous forme assertive et divisé en trois livres traitant successivement de phonétique, des parties du discours (traitées plus amplement que dans l'Ars minor) et des vices et figures, ce $3^{\mathrm{e}}$ livre contenant des bribes d'une syntaxe « en creux », c'est-à-dire traitée à l'occasion des défauts et figures.

4 Quant à la seconde, l'Ars Prisciani, auparavant nommée Institutiones grammaticae ${ }^{1}$, il s'agit d'un gros ouvrage dont on sait depuis toujours l'importance, comme en témoigne la transmission du texte (pensons aux 800 manuscrits existants), mais dont l'étude 
critique est plus tardive. Certes l'ouvrage constitue bien les vol. 2 et 3 du corpus des Grammatici Latini réuni par Keil à la fin du XIX ${ }^{\mathrm{e}}$ siècle, mais, comme nous avons pu le constater lors du colloque Priscien organisé à Lyon en octobre 2006 ( $c$ f. Baratin et al. éd. 2009), les études récentes sont moins nombreuses ${ }^{2}$. Par ailleurs, il n'existe pas encore de traduction "française" publiée de l'Ars de Donat, même si l'on sait que l'auteur de la monumentale édition du texte (1981), Louis Holtz, en a évidemment établi une, qui reste pour le moment inédite. Il ne faut pas oublier néanmoins qu'il existe bien des «traductions » dès le XIV siècle (cf. Colombo Timelli 1996 et Städtler 1988). Nous en verrons quelques exemples.

5 Naturellement, la tradition latine ne se limite pas à ces deux auteurs : il y a aussi toute la tradition médiévale à prendre en compte et il ne faut pas oublier que la tradition proprement "latine ", c'est-à-dire de description du latin en latin, continue à la Renaissance, avec un développement parallèle, qu'il ne faut pas négliger.

6 Ce que nous voudrions essayer de faire ici, c'est de rapprocher aussi systématiquement que possible les textes latins et les textes qui en sont issus pour décrire le français.

\subsection{Approche externe}

7 L'approche peut - et doit - être interne. Il faut rapprocher et comparer les textes euxmêmes. C'est pour l'essentiel ce que nous essaierons de faire dans cet article. Mais on peut aussi procéder à une approche externe, en relevant les citations des grammairiens latins dans le Corpus des grammaires françaises de la Renaissance. Dans les "auteurs sources d'exemples, de citation ou d'opinion ", Priscien apparait 19 fois et il est cité encore 13 fois dans les "autorités sur la langue sans citation", ce qui en fait le grammairien de l'Antiquité le plus mentionné devant Quintilien (18 et 4 fois) et Donat (3 et 4 fois).

8 Comment se fait la répartition? la majorité des citations du nom de Priscien sont chez Meigret (11) et Sylvius (19), les autres auteurs (Bosquet, Matthieu, Meurier, Ramus) ne le citant qu'une fois ou deux. Quant à Quintilien, il est cité sporadiquement par Matthieu, Pillot, Ramus, et Meigret, beaucoup plus massivement par Sylvius (16 fois). Enfin, en ce qui concerne Donat, son nom figure dans les titres mêmes de certaines œuvres, comme le montrent le Donait françois et le Aelii Donati de octo partibus orationis libellus, in dialogi formam Latinam et Gallicam redactus, avec son incipit: "Donatus \& Henricus de octo orationis partibus inter se colloquuntur. » De fait, Donat est tellement présent qu'il n'a pas besoin d'être cité: l'Ars Donati est encore au XVI ${ }^{\mathrm{e}}$ siècle la grammaire de référence qui constitue l'arrière-fond des méthodes pédagogiques. Donat est mentionné 4 fois par Sylvius et 2 par Bosquet, mais Meigret ne le cite pas.

9 La plus grande partie de la production grammaticale de l'époque est constituée d'ouvrages à vocation didactique, à l'intention des étrangers qui veulent apprendre le français, et leurs auteurs ne citent pas, ou citent peu, ou encore citent de façon vague ces illustres prédécesseurs. Par exemple, Pillot, le grammairien dont la méthode a eu le plus de succès si l'on en croit le nombre d'éditions, conseille simplement, pour les définitions des parties du discours - où l'on s'attend à voir reprises les définitions des grammairiens anciens - de consulter « les grammairiens latins, grecs et hébreux » (cf. infra).

10 Les deux textes qui citent le plus volontiers les grammairiens anciens sont ceux de Sylvius qui nomme presque autant Quintilien que Priscien, et de Meigret, très 
certainement parce qu'ils sont plus orientés vers la description théorique que vers l'apprentissage d'une langue étrangère. Meigret, s'il ne cite qu'une fois Quintilien, se place explicitement sous l'autorité de Priscien, comme le montrent ces mots qui terminent son texte :

Voilà donc les considérations que j'ai pu trouver des parties nécessaires au bâtiment de notre langage : lesquelles j'ai dressées suivant l'expérience que j'ai de notre langue et de son usage à l'imitation de l'ordre que tient Priscien en la latine. (Meigret 1980 [1550] : 141, [59], § 13)

\section{La place du latin dans la typologie des ouvrages}

\subsection{Tentative de typologie}

On tentera une typologie des grammaires de FLE, en fonction de la langue cible et de la métalangue. Il se trouve en effet que pour décrire le français, on a beaucoup utilisé le latin. Dans le Corpus des grammaires de la Renaissance, sur 21 ouvrages :

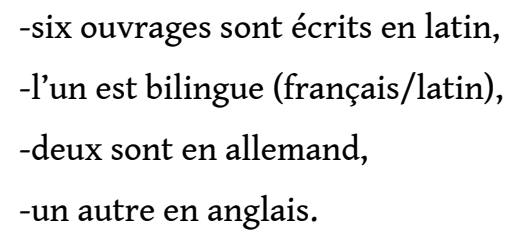
ouvrages sont à intention pédagogique, et le latin est choisi parce que c'est la langue de l'enseignement, notamment dans les régions germanophones, très friandes d'apprentissage de la langue française.

Ceci dit, notre Corpus comporte des grammaires écrites en français ou en bilingue qui ont pour cible non le français, mais le latin, et qui ne décrivent le français que pour faire comprendre la langue cible.

18 La nature de la métalangue est-elle importante par rapport à la langue décrite ? cela semble évident. Décrire une langue à travers le matériel élaboré pour une autre n'est pas sans importance. Néanmoins, le métalangage élaboré pour la langue initiale ne suffit pas forcément à décrire tous les phénomènes de la nouvelle langue à décrire : les grammairiens sont parfois obligés de recourir à des éléments qui n'appartiennent pas au métalangage traditionnel.

Donnons un exemple : pour la désignation du tréma utilisé par les imprimeurs français, les grammairiens utilisent certes des termes latins, mais pas forcément des termes reçus dans l'usage grammatical, simplement parce que, pour la description du latin, on n'en a pas eu besoin.

Désignation du tréma :

Sylvius : punctis duobus, velut apiculis ; apiculi gemino

Pillot: duobus apiculis; notatur in apice duobus punctis

Cauchie : geminis apicibus; duorum apicum usus

Serreius : duo apiculi ${ }^{3}$

21 Les premières grammaires pédagogiques du français sont donc écrites la plupart du temps en latin, parfois dans la langue de l'apprenant. Y a-t-il au XVI ${ }^{\mathrm{e}}$ siècle des grammaires françaises qui ne sont pas des grammaires de FLE? On pourrait répondre : oui, les grammaires en français, et on peut penser notamment aux grammaires de 
Meigret et de R. Estienne, on y reviendra. Mais ce ne serait pas vrai pour la plus ancienne grammaire du français en français, le Donait françois, attribué à John Barton, puisqu'elle a pour objet de perfectionner des Anglais en français. C'est donc bien une grammaire de FLE. Reprenons les ouvrages dans cette optique.

\subsection{Le cas de Sylvius, Meigret, Estienne et Ramus}

L'Isagwge et la Grammatica Latino-Gallica ne constituent peut-être pas une grammaire de FLE, encore que, dans la partie initiale, le mot grec eisagôgê signifie bien « introduction, initiation" à la langue française et que s'il y a des gens à introduire à la langue française, ce sont des étrangers : les Français eux-mêmes n'en ont pas besoin.

Latinè autem sum rem exequutus, vti hæc Anglis, Germanis, Italis, Hispanis, ac reliquis gentibus externis linguam Latinam non omnino ignorantibus, sermonis nostri ratio communis fieret. (Sylvius $1531: \mathrm{f}$. [a vir])

[C'est en latin que j'ai exposé ce sujet pour que le système de notre langue fût accessible aux Anglais, aux Allemands, aux Italiens, aux Espagnols et à toutes les autres nations étrangères qui n'ignorent pas tout à fait la langue latine.] (trad. Demaizière 1998 : 202 ; voir Chevalier 1994 : 18)

Néanmoins reconnaissons qu'écrire en latin pour faire comprendre aux étrangers le «système » de la langue française, ce n'est pas tout à fait écrire une grammaire pratique pour les étrangers. Les termes mêmes Grammatica Latino-Gallica présupposent qu'on peut écrire une grammaire valable pour les deux langues, et tout le texte insiste sur cette transformation du latin dans ce qui est devenu le français.

Meigret a pour objectif d'introduire les Français à une meilleure connaissance de leur langue, pour que tout à la fois, ils la parlent et l'écrivent mieux. Le terme François apparaît dès la première phrase, alors que le terme "étranger " apparaît trois fois seulement dans son Traité.

Combien que d'une pauvre considération la plus grande partie de nos Français soit en fantaisie que la poursuite d'une grammaire soit trop difficile et presque impossible en notre langue : je n'en ai pas pourtant si désespéré que je n'aie fait quelque diligence d'en chercher quelques moyens et règles. (Meigret, Aux lecteurs, 1980 [1550], p. 1)

Robert Estienne fait un Meigret plus clair. Mais un an après sa parution, donc en 1558, sa grammaire est traduite en latin par son fils, "pour servir aux étrangers désireux d'apprendre notre langue ", toujours avec la marque de l'olivier, puis réimprimée en 1560 chez André Wechel (Demaizière éd. 2003, introd. : 11). Sans être une grammaire de FLE, le Traicté d'Estienne a donc bien servi, au moins dans sa traduction latine, aux étrangers et, quand ils étaient en mesure de lire le français, aux «plusieurs desirans auoir ample cognoissance de nostre langue Francoise » (1557, au lecteur : 3 ).

Ramus évoque "vne grace et doulceur du Francoys, qui inuite les estrangers a lapprendre aussi curieusement, que nous apprenons en nos escolles le Grec et Latin » (1572, Preface à la Royne, p. [16]), mais sa grammaire est surtout faite pour répandre le bon français dans les autres disciplines :

Quelle aprenne a parler Francoys a ses compaignes, Rhethorique, Dialectique, Arithmetique, Geometrie, Musique, Astrologie, Physique, Ethique, Politique, par ainsi quelle ouure le pas aulx arts liberaulx pour retourner de Grece, et ditalie en la Gaulle. (Ramus, 1572, Preface à la Royne : [16-17] ; cf. Chevalier 1994 : 23) 
Est à noter également le cas des grammaires pour Flamands et Wallons, comme celles de Meurier (1557) et de Bosquet (1586) : elles peuvent être en français, car elles servent à un double usage: apprentissage pour les néerlandophones en contact avec les Wallons et donc pas complètement ignorants du français, perfectionnement pour les autres (cf. Bosquet, éd. Demaizière, $2005: 13)$. De Clercq (2000: 262) remarque que « la métalangue utilisée par Meurier se compose d'un mélange de latin et de vernaculaire ».

\subsection{Les grammaires pour les Anglais}

On a précisé l'objet du Donait françois; son titre même semble indiquer ce dont il est redevable à Donat, mais cette vision d'un Donait françois complètement calqué sur l'Ars Donati doit être fortement nuancée (cf. Colombo Timelli 2012) : l'ouvrage doit certes beaucoup à la tradition latine, mais bien plus à Priscien qu'à Donat (cf. Colombat 2013 et notre édition à paraître).

On attendrait de la grammaire de Palsgrave, écrite en anglais, qu'elle soit plus indépendante de la tradition gréco-latine. C'est vrai : les critiques (Baddeley, Chevalier) ont montré son originalité, la sensibilité à certaines tournures de l'anglais. L'influence latine n'est néanmoins pas complètement absente : le terme latin apparaît 32 fois dans le métatexte, Palsgrave faisant allusion à la latin tong(e), aux latin wordes, à la latin analogie, aux latin VIII partes of speche, aux latin grammariens. Et le métalangage reste en grande partie latin, comme en témoignent les indicateurs de conjugaison, par ex. au début de la table des verbes :

tercie coniugationis, prime coniugationis, secunde coniugationis, verbum medium prime coniugationis [normalisé par Génin : prim. conj. ou prim. conjug. dont il y a plus de 4000 occurrences ; l'abréviation de ter. conj. apparaît 242 fois] (1530 : c.xxxvi)

Il est vrai que ce métalangage latin est passé aussi facilement, voire plus facilement en anglais - declinatio > declination, coniugatio > coniugation, mais aussi secunda > seconde qu'il n'est passé en français ${ }^{4}$. Il est à noter également que, tout latin qu'il soit, medium appliqué au verbe (412 occurrences de verbum medium), qui correspond à l'anglais mean et qui désigne les verbes employés à la voix pronominale (Mon cueur se abhomine, or tout le cueur me fremyt), n'est pas un terme reçu de la terminologie latine traditionnelle.

\subsection{Les grammaires pour les Allemands}

C'est principalement dans ces ouvrages, ceux de Pillot, Garnier, Cauchie, Serreius, que le latin est la métalangue. Le lecteur d'aujourd'hui pourrait en conclure que ce sont des ouvrages savants. Comme on l'a déjà dit, ce serait un contresens : le latin est tout simplement la langue de l'enseignement, et comme l'explique Pillot, à l'issue d'une journée de cours, en latin, la récréation consiste en cet apprentissage du français, en latin, "mais en sorte que la chose se fasse seulement aux heures perdues, et qu'on n'enlève pas aux études principales les moments les plus propices » (Sed ita, vt succisiuis tantùm horis id fiat: et grauiora studia melioribus non defraudentur, Pillot 1561 [1550] / $2003: 4 / 6)$.

Pourquoi le latin? Lisons là aussi la réponse de Pillot :

Præterquam quòd manca sunt et imperfecta, non tam Gallicis ediscendis conferunt, quàm interpretandis Latinis [...]. Habeant sanè isti suum aliquem vsum, sed ad Gallicum sermonem cognoscendum parum iuuant. Adde quòd Gallicis isti verbis 
vtuntur, vt à suis tantùm videri possint, cùm meo iudicio, scribentem de aliqua re institutionem, oporteat exterorum et imperitorum præcipuam habere rationem. Neque enim qui Hebræas, Græcas, aut Latinas Grammaticas componunt, Hebraïcè, Græcè, aut Latinè scientibus, sed potiùs scire cupientibus componunt. (Pillot 1561 [1550] : 5-6).

[Outre que [les ouvrages existants] sont incomplets et imparfaits, ils ne contribuent pas tant à apprendre le français qu'à traduire les auteurs latins [...]. Je reconnais que ces ouvrages ont chacun leur utilité, mais ils aident trop peu à la connaissance de la langue française. Ajoutons que ces gens utilisent des mots français, en sorte que leurs ouvrages ne peuvent être vus que de leurs compatriotes, alors que, à mon avis, celui qui écrit une institution sur quelque sujet que ce soit doit tenir avant tout compte des étrangers et des novices. Et en effet ceux qui composent des grammaires hébraïques, grecques ou latines ne les composent pas pour ceux qui savent l'hébreu, le grec ou le latin, mais plutôt pour ceux qui désirent connaître ces langues.] (trad. Colombat, $2003: 8$ )

Les arguments de Pillot:

1. les grammaires françaises en français, outre qu'elles sont incomplètes et imparfaites, sont moins des ouvrages d'apprentissage du français que des ouvrages d'aide à la traduction ;

2. une "institution » est un ouvrage élémentaire, destiné aux débutants, et ceux-ci, pour comprendre, ne peuvent être formés directement dans la langue cible qu'ils ne connaissent pas encore.

34 La langue neutre ou «transparente ", celle qui sert à la communication, celle qui est outillée, c'est le latin, qui est utilisé pour la recherche de l'efficacité. C'est cette même recherche qui conduit à l'élaboration d'une grammaire différentielle : il s'agit de voir ce qui change, et seulement ce qui change, l'idée la plus répandue étant d'ailleurs que pas grand chose ne change. Ainsi, ce n'est pas la peine de donner les définitions des parties du discours :

Harum definitiones ex Latinis cognitas, hic percensere superuacuum putaui. (Sylvius $1531: 90$ )

[Leurs définitions [celles des parties du discours] étant connues d'après les Latins, j'ai jugé inutile de les passer en revue ici.] (trad. Demaizière, 1998 : 305)

Partes orationis, siue dictionum species, et differentiæ sunt octo. Harum partiúm definitiones, data opera prætermittimus, sicut etiam alia multa, quæ passim apud Grammaticos Latinos, Græcos, et Hebræos habentur : ad quos, si qui alicuius verbi descriptionem desiderabunt, remitto. (Pillot 1561 [1550] : 31)

[Les parties du discours, ou espèces de mots, ou catégories spécifiques, sont au nombre de huit [..]. Nous passons délibérément sous silence les définitions de ces parties du discours, tout comme beaucoup d'autres choses qui sont traitées chez les grammairiens latins, grecs et hébreux. Je renvoie à ces derniers qui désirera avoir la description d'un mot quelconque.] (trad. Colombat 2003 : 51)

Cette approche différentielle, qui exclut volontairement ce qui est déjà connu, se trouve aussi chez Garnier qui commence ainsi sa grammaire, excluant de traiter de la partie phonétique sous le prétexte qu'elle s'apprend par l'usage :

Superuacaneum iudicaui, hìc in limine multa tractare de Literis, Syllabis, aut Diphthongis. Vsus enim (qui omnium artium magister est) frequens et assidua lectio hæc omnia facilè indicabunt. (Garnier, $1558: 1$ )

[J'ai jugé superflu d'exposer beaucoup de choses ici, pour commencer, concernant les lettres, les syllabes et les diphtongues. En effet, l'usage fréquent (qui est maître de toutes sciences) et la lecture assidue révéleront facilement tout cela.] (trad. Cullière 2006:11) 
et qui pose parfois une homogénéité excessive, donc dangereuse, entre latin et français, ainsi au début du développement sur le pronom :

Pronomina apud Gallos ea ipsa sunt quæ et apud Latinos, declinationésque, casus, genera et numeros recipiunt, quemadmodum et nomina. (Garnier $1558: 23$ )

[Les pronoms chez les Français sont les mêmes que chez les Latins et ils reçoivent les déclinaisons, les cas, les genres et les nombres de la même manière que les noms.] (trad. Cullière $2006: 42$ )

\section{Les parties du discours}

On n'abordera pas ici les questions, pourtant importantes, du nombre des parties $\mathrm{du}$ discours et de la reconnaissance de l'article. On se focalisera sur le traitement du nom, avec, notamment, l'opposition substantif et adjectif, et sur le traitement du pronom. notable que pragma (action) est remplacé par res (chose), qui, faisant couple avec corpus (corps), orientera tout différemment la conception qu'on se fera du nom au Moyen Âge (voir Grondeux 2007).

L'adjectif n'apparaît que dans l'Ars maior, comme sous-classe, très basse dans la hiérarchie, de noms appellatifs, qui se place après les patronymica, les possessiua :

Sunt alia mediae significationis et adiecta nominibus, ut magnus, fortis; dicimus enim magnus uir, fortis exercitus; haec etiam epitheta dicuntur. (Donat, Ars maior: 616.7-8 H.)

[passage suivi par: "Sunt alia qualitatis, ut bonus, malus; alia quantitatis, ut magnus, paruus ; alia gentis (...), alia patriae (...).»]

[Il y a d'autres noms de signification intermédiaire et qui sont ajoutés à des noms, comme grand, courageux; nous disons en effet grand homme, armée courageuse; on les appelle aussi épithètes.]

La situation est beaucoup moins simple chez Priscien dans la mesure où la définition du nom y a rapport avec les notions de substance et d'accidents, dont la qualité : la combinatoire des concepts ainsi mis en jeu est complexe (pour une analyse détaillée, voir Garcea 2009). Certes, dans la définition donnée du nom au livre 2 : 
Nomen est pars orationis, quae unicuique subiectorum corporum seu rerum communem uel propriam qualitatem distribuit. (Priscien, Ars, GL 2, 56.29-57.1)

[Le nom est une partie du discours qui assigne à chacun des corps ou des choses pris pour objet une qualité propre ou commune.]

on retrouve les concepts de corpus et res, de proprium et de commune,mais on y trouve aussi celui de qualitas qu'on a également, couplé à la substance, dans le passage préliminaire aux parties du discours dans lequel Priscien distingue ce qui est spécifique à chacune d'entre elles :

Proprium est nominis substantiam et qualitatem significare. (Priscien, Ars, GL 2, 55.6)

[Le propre du nom est de désigner la substance et la qualité.]

Cette définition fait couple chez Priscien avec celle du pronom qui ne désigne que la substance (cf. Baratin, 1989, p. 398). Mais ce n'est pas sur ce point que nous insisterons ici. L'adjectif, défini à deux reprises, occupe une place beaucoup plus importante que chez Donat :

Adiectiua autem ideo uocantur, quod aliis appellatiuis, quae substantiam significant, uel etiam propriis adici solent ad manifestandam eorum qualitatem uel quantitatem, quae augeri uel minui sine substantiae consumptione possunt, ut bonum animal, magnus homo, sapiens grammaticus, magnus Homerus. (Priscien, Ars, GL $2,58.20-24)$

[Les adjectifs sont ainsi appelés parce qu'ils s'ajoutent habituellement à d'autres appellatifs (noms communs) qui désignent la substance, ou même à des noms propres pour en manifester la qualité ou la quantité, lesquelles peuvent être augmentées ou diminuées sans que cela touche la substance, comme bon animal, grand homme, savant grammairien, grand Homère.]

Adiectiuum est, quod adicitur propriis uel appellatiuis et significat laudem uel uituperationem uel medium uel accidens unicuique: laudem, ut iustus; uituperationem, ut iniustus; medium, ut magnus (dicimus enim magnus imperator laudantes et magnus latro uel fur uituperantes). accidens uero, id est suum uniuscuiusque, ut niger coruus et altum mare. (Priscien, Ars, GL 2, 60.6-11)

[L'adjectif est ce qui est ajouté aux propres ou aux appellatifs et il indique la louange ou le blâme, ou bien une qualification ambivalente, ou bien un accident affectant un individu: la louange, comme juste, le blâme, comme injuste; une qualification ambivalente, comme grand - nous disons en effet grand général à titre de louange, et grand bandit ou grand voleur à titre de blâme -; quant à l'accident, c'est-à-dire ce qui est propre à un individu, c'est par exemple noir corbeau, mer profonde.]

Que devient cette organisation dans les premières grammaires du latin traduites en français? Cela dépend des ouvrages. Dans le Libellus, c'est la présentation traditionnelle de Donat, avec sa définition et la division en propre et commun :

H. Nomen quid est? Qu'est-ce que le Nom? D. Pars oratiônis cum casu, corpus aut rem, própriè, communitérve signíficans. Própriè, vt Roma, Tyberis : Commúniter, vt vrbs, flumen. C'est vne partie d'oraison auec le cas, signifiant proprement ou communeement vn corps, ou vne chose. Proprement, comme la ville de Rome, le Tybre: Communeement, comme vne ville, vn fleuue. (Libellus 1585 [1545] : 3).

alors que dans le traité Quantes parties, la définition est bien gardée, mais la division en " manières » introduit immédiatement l'opposition substantif / adjectif établie sur une base morpho-syntagmatique :

Qu'est nom? Une partie d'oroison qui senefie substance o qualité propre ou commune. Quantes manieres de nom sont? II. Queles ? Le substantif et li adjectif; le substantif qui se decline par une article si comme hic magister, ou par II au plus si comme hic et hec sacerdos, l'ajectif par III articles si comme hic et hec et hoc felix, ou 
par III diverses terminaisons si comme bonus, na, bonum. (Quantes parties:

f. $\left.41^{\mathrm{r} b}-41^{\mathrm{v} a}\right)$ traitée au titre des accidents, mais quand les auteurs abordent le nom, c'est maintenant l'opposition substantif $v s$ adjectif qui fait partie inhérente de la définition :

Coment cognoistrey je de celle partie de oraison qu'est appellé nom ? Chescun mot que porte le nom de une chose par soy mesmes ou pendant d'un aultre est appellé nom. Pour quoy ditez vous par soy mesmes? Pour ces noms que sont appellés substantifs, si come une femme, un home. Et pour quoy ditez vous pendant d'un aultre ? Pour les noms que sont appellés adjectifs, que ne povent pas estre par eulx, mais il leur fault tous jours ou estre aveque leurs substantifs ou les avoir entenduz, si come bon, bel. (Donait françois :f. $319^{\mathrm{r}} \mathrm{a}$ )

Cette définition globale qui définit très clairement à la fois le nom, le substantif et l'adjectif constitue un cas particulier. Dans la plupart des ouvrages du XVI ${ }^{e}$ siècle, notamment les ouvrages pédagogiques, il y a beaucoup plus d'implicite. Ainsi ne trouve-t-on de définition du nom ni chez Palsgrave, ni chez Sylvius, ni chez Pillot, ni chez Garnier, ni chez Cauchie, ni chez Serreius. Chez Cauchie, le passage sur le nom commence ainsi :

DE NOMINE. Adiectiuum quando solum subsistit neque adiacet cuiquam substantiuo naturam substantiui induit, et tum sibi uel uoculam un uel articulum præire patitur : talia sunt un exemplaire ab adiectiuo lat. exemplaris [...]. (Cauchie, 1586, f. $15 \mathrm{v})$

[LE NOM. L'adjectif, quand il demeure seul et ne s'ajoute pas à un substantif, revêt la nature du substantif et supporte alors que le petit mot un ou un article le précède, tel sont un exemplaire, de l'adjectif latin exemplaris [...] (trad. Demaizière 2001 : 321-322) Voyons maintenant l'articulation des divisions :

51

chez Palsgrave, on trouve uniquement l'opposition substantif vs adjectif ; l'opposition nom propre vs nom commun n'est pas évoquée ; proper name(s) n'apparaît que sept fois dans les 1000 pages du texte (donc 4 occurrences peu significatives dans les listes de noms), et n'est jamais opposé à un «nom appellatif» ou «commun» (alors qu'on a commyn gender dans le texte);

52 - chez Sylvius, qualité des noms (propres et appellatifs), substantif et adjectif apparaissent sans être définis ;

53 - Pillot ajoute dans l'éd. de 1561 une remarque (qui ne figure pas dans l'éd. de 1550) sur l'inutilité de la division propre $v s$ appellatif :

Priore enim illa partitione, qua nomen diuidunt in proprium et appellatiuum, non opus habemus. (1561:34)

[Nous n'avons pas besoin de cette première partition par laquelle on divise le nom en propre et appellatif.]

54 - Garnier oppose propre vs appellatif, qui lui-même se subdivise en substantif vs adjectif, "ce qui revient à dire que le nom propre n'est pas substantif » (Cullière $2006: 23$, note 2 ) ;

55 - chez Cauchie, il y a un grand usage implicite de appellatif vs propre, de substantif vs adjectif, sans que ce soit commenté.

56 Intéressons-nous maintenant aux auteurs qui donnent une définition. Comme attendu, Meigret propose une définition du nom traduite de Priscien : 
Combien que toutes paroles et vocables, tant verbes, adverbes que prépositions et autres parties du langage puissent être appelés noms : nous considérons toutefois ici le nom de tant qu'il est une partie du langage ou oraison signifiant la propre ou commune qualité de toutes choses. (Meigret 1980 [1550] :23, [8], § 1)

57 Fait remarquable : il n'y a pas chez Meigret de subdivision initiale entre substantif et adjectif, ni de définition du substantif, sans doute parce que chez Priscien, il n'y a que le verbe qui peut être substantif. En revanche, on trouve une définition de l'adjectif quasiment traduite de Priscien, la catégorie «intermédiaire » (medium) étant traduite simplement par « le moyen ».

Toutes les autres espèces presque se trouvent es noms appellatifs : comme l'adjectif, lequel on conjoint aux noms propres et appellatifs, et a signification de blâme ou louange ou de moyen : ou de quelque accident : comme bon, juste pour la louange [le lós] : et pour le blâme maovęs, injuste : pour le moyen grant, exçellęnt : car nous disons un grant home de bien et un gran' larron: là où grant sert à l'un de vitupère : et à l'autre de louange. Quant à l'accident qui est le propre d'une chacune chose, nous disons le corbeao noẸr, la nEje blanche. (Meigret 1980 [1550] : 25, [8], § 10)

Chez R. Estienne, l'opposition substantif $v s$ adjectif précède celle de propre vs appellatif, et à la différence de Meigret, le substantif est défini : comme dans le Donait françois, sur un plan sémantique, le nom est défini par son autonomie et l'adjectif par sa dépendance :

Les Noms, sont les mots qui signifient vng corps ou chose qu'on peut toucher et veoir, comme, Liure, Arbre: ou chose qui ne peult estre touchee ne veue, comme Vertu, Esprit, Dieu.

Il y a deux sortes de Noms: les vns sont appelez Substantifs, desquels la signification est entendue sans qu'autres mots leur soyent adioincts. comme Pain, Terre : et font vng sens parfaict auec l'adiectif : comme Pain blanc, Terre noire. Les adiectifs sont les mots qui se mettent auec les substantifs pour declarer leur qualite ou quantite : et ne se mettent point proprement sans substantif, ou autrement on ne scauroit a quoy seruiroit ledict adiectif : comme en disant Blanc, tu ne peux rien entendre si tu n'adioins quelque substantif : comme disant, Pain blanc, Terre noire [...]. (R. Estienne $1557: 13$; éd. Demaizière, $2003: 39-40$ )

Dans l'optique taxinomique binaire de Ramus, nom, substantif et adjectif sont définis par leur combinaison avec un attribut spécifique : nom : nombre + genre ; substantif : un seul genre ; adjectif : des deux genres :

Le mot aian' nombre e' nom ou verbe. Nom et un mot de nombr' avec jenre. (Ramus $1562: 41)$

Or de la diferense du jenre, le nom e' sustantif ou ajectif : Sustantif, ci et d'un seul jenre, ajectif, ci et de l'un e l'autre. (Ibid. : 44)

Dans l'édition de 1572, l'adjectif n'est pas défini :

Lę mot dę nõbrę ẻt nom $\curlyvee$ vẻrbę. Nom s'ẻt vn mot dę nombrę avẻK jẻnrȩ.

Le mot de nombre est nom ou verbe. Nom cest vng mot de nombre auec genre.

(Ramus 1572: 58-59)

P. Dę la difẻrẻnsȩ du jẻnrȩ, lę nom ẻt sustantif $\gamma$ ajẻKtif. Sustantif, Ki ẻt d'un seul jẻnre.

P. De la difference du genre, le nom est substantif ou adjectif. Substantif, qui est dun seul genre. (Ibid. : 66-67)

61 Chez Bosquet, l'opposition entre substantif et adjectif n'est plus seulement sémantique (critère des degrés de comparaison) : elle est aussi syntaxique, avec une référence à l'autonomie ou à la dépendance dans l'oraison :

Qu'est-ce de Nom? - C'est vne partie d'oraison declinable, sans aucuns temps; laquelle signifie quelque chose ; ayant pour principaux indices-le, \& la. [...] 
Qu'est-ce de-Substantif? - C'est vn nom, quy deuant le verbe, peut consister en vne oraison, sans qu'il soit adioinct auec autre nom; et ne se compare nullement, comme-Poëte, qui est Substantif ; on peut dire en parfait sens -le Poëte enseigne. [...] Qu'est-ce de nom adiectif? - C'est vn nom, qui ne peut consister seul en vne oraison, sans estre adioinct auec vn substantif; car qui diroit le docte enseigne doctement; le sens ne seroit cõplet; mais il faut adioindre vn suppost, comme Poëte, Orateur, ou maistre, ainsy-le sçauãt maistre (ou Poëte) enseigne doctement, ainsy d'autres.

Et peut l'adiectif qui s'augmente, ou diminuë estre comparé par ses degrez. (Bosquet 1586 [1566 ?] : 42-52 : éd. Demaizière 2005 : 65-72) il y a deux traditions différentes, et nettement divergentes, l'une inspirée de Donat et l'autre de Priscien ( $c f$. Lagarde 1985). Cela tient à la définition, qui fait intervenir la notion de personne de deux façons différentes, et à l'extension de la classe et des items recensés.

Pour Donat, le pronom, qui peut être « défini » ou « indéfini » (l'Ars maior y ajoute les «moins que définis» ipse, iste), peut remplacer aussi bien un nom propre qu'un appellatif; il peut « quelquefois » (interdum) recevoir la personne.

Pronomen quid est? Pars orationis, quae pro nomine posita tantundem paene significat personamque interdum recipit. [...] Qualitas pronominum in quo est? Bipertita est : aut enim finita sunt pronomina aut infinita. Quae sunt finita? Quae recipiunt personas, ut ego, tu, ille. Quae sunt infinita ? Quae non recipiunt personas, ut quis quae quod. (Donat, Ars minor : 588.2-7 H.)

[Qu'est-ce qu'un pronom? une partie du discours qui, mise à la place d'un nom, signifie à peu près la même chose et reçoit quelquefois la personne. En quoi consiste la qualité des pronoms. Elle se divise en deux : soit les pronoms sont définis, soit ils sont indéfinis. Quels sont ceux qui sont définis? ceux qui reçoivent la personne, comme je, tu, il. Quels sont ceux qui sont indéfinis? ceux qui ne reçoivent pas la personne, comme qui.]

Il en résulte une liste longue établie dans l'Ars maior (finita, infinita, minus quam finita, praepositiua, subiunctiua, gentis, ordinis, etc.) à laquelle s'ajoutent des items, comme neuter ou uter sur le statut desquels (pronoms ou noms) on peut, selon Donat, hésiter.

$65 \mathrm{Au}$ contraire, pour Priscien, le pronom remplace toujours un nom propre et une personne déterminée :

Pronomen est pars orationis, quae pro nomine proprio uniuscuiusque accipitur personasque finitas recipit. (Priscien, Ars, 12, 1 GL2, 577.2-3)

[Le pronom est une partie du discours qui est admise à la place du nom propre de chacun et qui reçoit des personnes définies.]

Il en résulte une liste beaucoup plus courte (8 primitifs : ego, tu, ille, ipse, iste, hic, is, sui ; 7 dérivés : meus, tuus, suus, noster, nostras, uestras) et surtout, Priscien prend bien soin, au livre 2, d'expliquer l'exclusion des indéfinis de Donat :

Proprium est pronominis pro aliquo nomine proprio poni et certas significare personas. Ergo quis et qui et qualis et talis et quantus et tantus et similia, quae sunt infinita siue interrogatiua uel relatiua uel redditiua, magis nomina sunt appellanda quam pronomina : neque enim loco propriorum nominum ponuntur neque certas significant personas, sed etiam substantiam, quamuis infinitam, et qualitatem, 
quamuis generalem, quod est suum nominis, habent : nomina sunt igitur dicenda, quamuis declinationem pronominum habeant quaedam ex eis. (2, 18 GL2, 55.13-20)

[Le propre du pronom est d'être utilisé à la place d'un nom propre et de désigner des personnes déterminées. Donc quis et qui, qualis et talis, quantus et tantus et les mots semblables, qui sont indéfinis, interrogatifs, relatifs [anaphoriques] ou corrélatifs, doivent être appelés plutôt des noms que des pronoms. Ils ne sont en effet pas mis à la place de noms propres et ne désignent pas des personnes déterminées, mais en outre ils ont une substance, bien qu'indéfinie, et une qualité, bien que générale, ce qui est le propre du nom : ils doivent donc être appelés des "noms", bien que certains d'entre eux aient une déclinaison de pronom.]

Qu'en est-il dans nos grammaires de la Renaissance ? Là aussi, il n'y a pas toujours de définition, ainsi chez Sylvius, Pillot, Cauchie. Mais chez Sylvius, on remarquera que quis/qui est bien placé parmi les noms.

Nomen præterea nobis, vt Græcis et Latinis, est multiplex. Gentile, vt Parrhisiensis Parrhisien. Infinitum, vt quis, quøe, qui [...]. (Sylvius 1531 : 98)

[En outre, pour nous, comme pour les Grecs et les Latins, le nom est multiple : nom d'origine, comme Parrhisiensis Parrhisien; indéfini, quis, quae, qui [...].] (trad. Demaizière 1998 : 313)

ce qui n'empêche pas l'auteur d'en traiter aussi dans le développement sur les pronoms (Sylvius $1531: 112-113$ ).

Dans les traités qui ont pour objet la langue latine, le traitement varie : le traité Quantes parties suit Priscien, alors que le Libellus suit Donat:

Qu'est pronon? Une partie d'oroison qui est mise pour propre non et senefie certainne personne. (Quantes parties, f. $46^{\mathrm{v}} \mathrm{a}$ )

Pronômen quid est? Qu'est-ce que le Pronom? D. Pars oratiônis, quæ pro nómine pósita, tantúndem penè signíficat, personámque intérdum récipit, C'est vne partie d'oraison, laquelle mise pour le nom, signifie presque tout autant que le nom, et aucunesfois reçoit personne. (Libellus, 1585 [1545] : 7)

\section{Le Donait françois suit Priscien :}

Coment cognoistrey je un pronom? Chescun mot que est mis en lieu d'un nom et oveques ce seignifie ${ }^{7}$ certeine personne est appellé un pronom. (Donait françois: f. $319^{\mathrm{r} b}$ )

avec une conséquence importante sur le classement des noms adjectifs, où l'on trouve un certain nombre d'éléments qui étaient traités comme des pronoms dans la tradition donatienne :

Quantz maniers est il des adjectifs? Six. Quielx? Interogatif, si come combien; demonstratif, si come bon; distributif, si come chescun; partitif, si come aucun; infinitif, si come quelque; negatif, si come nul. (Ibid., dans le passage traitant du nom, juste avant)

72 Palsgrave établit une classe large de pronoms sur le modèle de Donat, mais il y a deux passages qui manifestent une hésitation sur la nature de certains d'entre eux: dans l'introduction au second livre, après avoir évoqué les catégories principales que sont les primitifs, les dérivatifs et les démonstratifs, Palsgrave ajoute :

We may also contayne under the pronowne other thre dyvers sortes relatyves, as qui or lequel, interrogatyves, as qui, and numeralles as vng, deux, troys, etc. And I speke also amongest the pronownes in my seconde boke of nownes partityves and distributyves, as tout, nul, aulcun, quelqun, chascun, etc. (1852 [1530] : XXIX)

[Nous pouvons aussi inclure sous le pronom trois autres sortes de relatifs, tels que qui et lequel, des interrogatifs tels que qui, et des numéraux, tel que ung, deux, troys, etc. Je parle aussi, dans mon second livre, au chapitre des pronoms, des noms 
partitifs et distributifs, tels que tout, nul, aulcun, quelqun, chascun, etc.] (trad.

S. Baddeley 2003 : 343-344)

L'élargissement se fait en deux temps, avec d'abord les relatifs, les interrogatifs et les numéraux, et ensuite les partitifs et les distributifs, qui sont bien qualifiés de noms. Au second livre, remarquant que les pronoms se comportent comme les noms et doivent gouverner le verbe en sorte qu'il y ait un accord en nombre et en personne, il ajoute cette remarque qui annonce nos « adjectifs possessifs » :

Except pronownes derivatyves, whiche folowe rather the nature of adjectyves, and must nedes have some substantyve to be joyned vnto. (1852 [1530] : 74)

[Il faut en excepter les pronoms dérivés, qui sont plutôt de même nature que les adjectifs, et qui doivent être joints à un substantif.] (trad. S. Baddeley $2003: 451$ )

\section{Meigret propose cette définition :}

Le pronom est une partie du langage inventée pour suppléer le nom, tant propre qu'appellatif, sans aucune signification de temps, dénotant toujours quelque certaine personne. (Meigret 1980 [1550] : 49, [16], § 1)

dans laquelle "tant propre qu'appellatif » semble indiquer le parti pris pour Donat, alors que « dénotant toujours quelque certaine personne » vient bien de Priscien. On a donc un mixte. Il n'en reste pas moins que du point de vue de l'organisation générale, Meigret suit Donat, traitant du pronom après le nom, et rangeant dans les pronoms les relatifs, c'est-à-dire tous les anaphoriques ([21] §24-38), y compris qi dont il dit (réminiscence non complètement assumée de Priscien ?) qu'il est « le plus général» :

Nous avons encore d'autres relatifs desquels celui que nous appelons qi est le plus général: parce qu'il sert à tous genres, tous nombres et à toutes personnes. (Meigret 1980 [1550], p. 59, [21], § 30 \# Estienne, 1557, p. 27)

6 Mais Meigret ne donne pas de justification explicite à son reclassement. Hausmann (1980 : 170), commentant l'organisation du chapitre sur le pronom, dit que « le chapitre est loin d'être clairement bâti. On a quelque peine à comprendre que Meigret distingue, pour le moment, cinq sortes de pronoms: personnels, démonstratifs, relatifs, réciproques et possessifs ». Chez R. Estienne, fidèle épigone de Meigret, on retrouve à la fois la définition étroite de Priscien et la liste large de Donat :

Pronoms, est vne sorte de mots qui seruent pour supplier le Nom tant propre, qu'appellatif, sans aucune signification ou declaration de temps, denotant tousiours quelque certaine personne. [...]

Il y a douze Pronoms, Ie, Tu, Soy, ou Se, Il, Ce, Cest, Eulx, Mon, Ton, Son (ou Mien, Tien, Sien,) Nostre, Vostre. [...]

Nous auons encores d'autres relatifs, desquels celuy que nous appelons Qui, est le plus general, par ce qu'il sert a tous genres, a tous nombres, et a toutes personnes. (R. Estienne 1557, p. 21-27 / 2003, p. 49-56)

tandis que Ramus, toujours soucieux de simplification et de cohérence théorique, regroupe, à la suite de Sanctius, les catégories du nom et du pronom :

D. Cę ditę' vou' de' pronõs ? P. Di' nom' son' vulgerement apele' pronoms, e semblęt avoer celcęcazę. (Ramus $1562:$ 46-47)

Kę ditȩs v४s dẻs pronoms? P. Dis noms sont isi apȩles pronoms.

Que dictes vous des pronoms. P. Dix nõs sont icy appelles pronõs. (Ramus 1572 : 70)

Les pronoms ne sont que des noms qui ont une flexion spéciale. Les relatifs, il, luy, puis qui que en constituent les $5^{\mathrm{e}}$ et $6^{\mathrm{e}}$ catégories. 
79 À la fin du siècle, chez Bosquet, la définition de Priscien est toujours là, mais la liste est redevenue la liste large, intégrant les thèmes en qu- qui sont bien repassés dans les pronoms, comme chez Donat:

Qu'est-ce de Pronom? - C'est vne partie d'oraison, laquelle se met au propre lieu d'vn nom, et signifie certeine personne.

Y a-il plusieurs sortes de pronoms? - Ouy, de quatre sortes, à sçauoir, demonstratifs, Relatifs, possessifs, et Reciproques. (Bosquet, 1586 [1566 ?], p. 63)

\section{Conclusion} pourrait penser que les différences entre les langues sautent aux yeux: or le pédagogue, conditionné par un cadre descriptif différent, les voit sans doute moins aisément qu'on ne pourrait le croire. En tout cas, la tendance dominante, peut-être plus dans les grammaires pratiques, dont la tâche est avant tout de faciliter l'apprentissage, est de tenter de réduire ces divergences, en mettant en avant les similitudes. C'est de dire : "C'est la même chose ", sous-entendu: "C'est facile, puisque c'est la même chose ». Les Latins l'avaient fait par rapport au grec (cf. Desbordes 2007), nous ne sommes pas étonnés que les Français reproduisent le même schéma.

81 La recherche de la similitude entraine fatalement une attitude conservatrice : quand une catégorie est solidement installée dans votre tête, il est très difficile de penser en dehors d'elle. Cela a conduit à "sauver» les catégories reconnues pour la langue ancienne; dans le traitement des accidents, l'un des exemples les plus massifs et les plus prégnants est le maintien du cas dans le nom français jusqu'au XVII ${ }^{\mathrm{s}}$., voire audelà.

Du côté des parties du discours, face au nom, beaucoup de grammairiens croient être en territoire connu et n'éprouvent pas le besoin de le définir. Pourtant, la situation a complètement changé : l'opposition substantif / adjectif a pris le pas sur l'opposition antique nom propre/ nom commun. Les définitions du Donait françois, puisde R. Estienne, et même de Bosquet, sont complètement renouvelées par rapport à celles que donnent Donat et Priscien, les auteurs insistant sur une autonomie, à la fois sémantique et syntaxique, du substantif à opposer à la dépendance de l'adjectif, alors que d'autres auteurs, comme celui du traité Quantes parties (qui décrit le latin), mais aussi Ramus, rapportent la description à la simple expression des marques morphologiques.

83

Dans le cas du pronom, il y a, au départ, une forte différence de conception entre Donat et Priscien, avec, comme conséquence, une définition différente, mais aussi une répartition différente des items. Pourtant les conséquences ne semblent pas si lourdes pour peu que l'on ne s'attache pas trop aux contraintes théoriques: la définition inspirée de Priscien (le pronom exprime «une certaine personne») est majoritairement retenue, ce qui n'empêche pas les auteurs d'ajouter des pronoms à la liste de Priscien, et ainsi, notamment, de faire rebasculer les relatifs (refoulés dans les noms par Priscien) dans les pronoms. La problématique théorique initiale a été ainsi oubliée : ce n'est pas un exemple isolé, c'est même la situation la plus fréquente. 


\section{Bibliographie}

\section{Sources}

Les textes latins antiques (Donat, Priscien) sont accessibles sur le site du Corpus grammaticorum Latinorum

(http://htl2.linguist.jussieu.fr:8080/CGL/), A. Garcea \& V. Lomanto éd.

Les textes français cités sont empruntés au Grand Corpus des grammaires de la Renaissance, lui-même partie du Grand Corpus des grammaires françaises, des remarques et traités sur la langue (XIV ${ }^{e}-X V I{ }^{e}{ }^{e}$.), B. Colombat, J.-M. Fournier, W. Ayres-Bennett dir. Paris : Classiques Garnier Numérique. Les éditions papier utilisées sont celles de la série Traités sur la langue française (coll. Textes de la Renaissance), dir. C. Demaizière. Paris : Champion. Nous avons exploité les textes suivants :

[DONAT]. Quantes parties d'oraison sont? $14^{\mathrm{e}} \mathrm{s}$.

[BARTON, Johan]. Donait françois, ca. 1409. Éd. E. Stengel (1879), P. Swiggers (1985), T. Städtler (1988), B. Colombat (à paraître).

John PALSGRAVE. Lesclarcissement de la langue françoyse, Londres, 1530. Éd. F. Génin (1852). Éd. S. Baddeley, 2003.

Jacobus SYLVIUS Ambianus [Jacques Dubois]. In linguam Gallicam isagwge et Grammatica LatinoGallica, Paris, 1531. Éd. C. Demaizière, 1998.

Aelii Donati de octo partibus orationis libellus, Paris, 1585 [1545].

Louis MEIGRET. Le trĘtté de la grammĘre françoĘze, Paris, 1550. Éd. F.-J. Hausmann, 1980.

Jean PILLOT. Gallicae linguae institutio, Latino sermone conscripta, Paris, 1561 [1550]. Éd. B. Colombat, 2003.

Robert ESTIENNE. Traicté de la grãmaire Francoise, Paris, 1557. Éd. C. Demaizière, 2003.

Gabriel MEURIER. La Grammaire françoise, Anvers, 1557. Éd. C. Demaizière, 2005.

Jean GARNIER. Institutio gallicae linguae in usum iuuentutis Germanicae, Genève, 1558. Éd. A. Cullière, 2006.

Antoine CAUCHIE. Grammaticae gallicae libri tres, Strasbourg, 1586 [1570]. Éd. C. Demaizière, 2001.

Petrus RAMUS [Pierre de la Ramée]. GramerĘ, Paris, 1562.

Petrus RAMUS [Pierre de la Ramée]. Grammaire, Paris, 1572. Éd. C. Demaizière, 2001.

Jean BOSQUET. Elemens ou institutions de la langue françoise, Mons, 1586. Éd. C. Demaizière, 2005. Joannes SERREIUS [Jean Serrier]. Grammatica Gallica nova, Strasbourg, 1623 [1598]. Éd. A. Jacquetin, 2005.

\section{Éléments de bibliographie secondaire}

AUROUX, Sylvain (1994). La révolution technologique de la grammatisation. Liège : Mardaga. 
BARATIN, Marc (1989).La naissance de la syntaxe à Rome. Paris : Éd. de Minuit. BARATIN, Marc, COLOMBAT, Bernard, HOLTZ, Louis éd. (2009). Priscien, Transmission et refondation de la grammaire, De l'Antiquité aux Modernes. Turnhout : Brepols (Studia Artistarum 21).

CHEVALIER, Jean-Claude (1994). Histoire de la grammaire française. Paris : PUF (Que sais-je? $\left.n^{\circ} 2904\right)$.

COLOMBAT, Bernard (1999). La grammaire latine en France, à la Renaissance et à l'Âge classique. Théories et pédagogie. Grenoble : ELLUG.

COLOMBAT, B. (2011). « Les Grammatici Latini vus depuis la Renaissance et le XVI ${ }^{\mathrm{e}}$ siècle : les définitions du nom ", in N. N. Kazansky, V. I. Mazhuga, I. P. Medvedev, L. G. Stepanova †, P. Swiggers, A. Wouters (eds.), Ancient Grammar and its posterior tradition, St. Petersburg Colloquium (April 20-23, 2005), Leuven, Peeters (O/S 36), p. 227-247.

COLOMBAT, B. (2013). « Le Donait françois est-il un vrai Donat?». In S. Große, A. Hennemann, K. Plötner, S. Wagner (Hrsg./éd.). Angewandte Linguistik. Linguistique appliquée. Zwischen Theorien, Konzepten und der Beschreibung sprachlicher Äußerungen. Entre théories, concepts et la description des expressions linguistiques. Frankfurt am Main : Peter Lang, 31-46.

COLOMBAT, B. (à par.). [John Barton]. Donait françois, édition commentée. Paris : Classiques Garnier.

COLOMBAT, B. éd. (1992). « L'adjectif : perspectives historique et typologique ». Histoire Épistémologie Langage 14/1 et Archives et documents de la SHESL, seconde série, 6.

COLOMBO TIMELLI, Maria (1996). Traductions françaises de l'Ars minor de Donat au Moyen Âge (XIII ${ }_{-}$ $X V^{e}$ siècles). Firenze : La Nuova Italia.

COLOMBO TIMELLI, Maria (2012). « Ruptures ou Continuité ? La grammaire de matrice donatienne au siècle de la Renaissance ». In B. Colombat, J.-M. Fournier et V. Raby éd.. Vers une histoire générale de la grammaire française. Matériaux et perspectives. Paris : Champion, 97-114.

DE CLERCQ, Jan, LIOCE, Nico, SWIGGERS, Pierre éd. (2000). Grammaire et enseignement du français, 1500-1700. Leuven : Peeters (O/S 16).

DE CLERCQ, Jan (2000). « La grammaire française (1557) de Gabriel Meurier ». In De Clercq, J. et al. éd., 237-276.

DE NONNO, Mario (2009). «Ars Prisciani Caesariensis : problemi di tipologia e di composizione ». In M. Baratin, B. Colombat, L. Holtz éd., 249-278.

DESBORDES, Françoise (2007). Idées grecques et romaines sur le langage. Travaux d'histoire et d'épistémologie. Lyon : ENS Éditions.

GARCEA, Alessandro (2009). « Substance et accidents dans la grammaire de Priscien ». In M. Baratin, B. Colombat, L. Holtz éd., 125-138.

GRONDEUX, Anne (2007). « Res meaning a thing thought : the Influence of the Ars Donati ». In J. Marenbon ed., The Traditions of Ancient Logic in the Middle Ages. Vivarium, 45, 189-202.

GROUPE ARS GRAMMATICA (2010). Priscien, Grammaire, livre XVII, Syntaxe, 1, texte latin, traduction introduite et annotée. Paris : Vrin.

LAGARDE, Jean-Pierre (1985). « L'influence de Donat et de Priscien sur la description du pronom au XVI ${ }^{\mathrm{e}}$ siècle ». Verbum, De la plume d'oie à l'ordinateur, études de philologie et de linguistique offertes à Hélène Naïs, 263-275.

LALLOT, Jean (1998). La grammaire de Denys le Thrace, traduite et annoté. Paris : Vrin. 
ROSIER, Irène (1992). «Quelques aspects de la diversité des discussions médiévales sur l'adjectif ». In B. Colombat éd. Histoire Épistémologie Langage, $14 / 1,75-100$.

STÄDTLER, Thomas (1988). Zu den Anfängen der französischen Grammatiksprache. Textausgeben und Wortschatzstudien. Tübingen : Niemeyer.

SWIGGERS, Pierre (1988). « Les premières grammaires des vernaculaires gallo-romans face à la tradition latine : stratégies d'adaptation et de transformation ». In I. Rosier dir. L'héritage des grammairiens latins de l'Antiquité aux Lumières. Leuven : Peeters, 259-269.

SWIGGERS, P. (1997). « Le TrĘttè de la grammĘre francoĘze (1550) de Louis Meigret : la description et la terminologie du nom ». In M. Lieber \& W. Hirdt (Hrsg.). Kunst und Kommunikation, Betrachtungen zum Medium Sprache in der Romania, Festschrift zum 60. Geburtstag von Richard Baum. Tübingen : Stauffenburg Verlag, 311-325.

SWIGGERS, P. (2001). « La terminologie et la description du pronom dans la grammaire française du seizième siècle ». In B. Colombat \& M. Savelli éd. Métalangage et terminologie linguistique. Leuven : Peeters (O/S 17), vol. 1, 395-412.

SWIGGERS, P. (2003). «Continuités et discontinuités, tension et synergie : les rapports du latin et des langues vernaculaires, reflétés dans la modélisation grammaticographique ». In M. Goyens \& W. Verbeke eds. The Dawn of the Written Vernacular in Western Europe. Leuven : Leuven University Press, 71-105.

SWIGGERS, P. \& VANHOECKE, Willy éd. (1989). La langue française au XVI ${ }^{e}$ siècle : usage, enseignement et approches descriptives. Louvain : Leuven University Press / Peeters.

\section{NOTES}

1. Institutiones grammaticae est le titre traditionnel; les travaux de M. De Nonno (notamment 2009) ont montré que le terme de Ars (grammatica) est plus conforme à la dénomination originelle.

2. Il existe néanmoins des travaux d'établissement du texte en Italie, une traduction allemande en cours, par Axel Schönberger ; une traduction française est également en cours, par le groupe Ars grammatica, animé par Marc Baratin : le premier volume, consacré au livre 17 (le premier livre de la syntaxe) est paru en 2010, le second, comportant les livres 14-16 (préposition, adverbe, conjonction), est paru en 2013 ; le livre 18 (deuxième partie de la syntaxe) est en cours de traduction.

3. Données extraites de notre communication à la 10e journée d'étude des grammaires françaises de l'âge classique, journée consacrée à la ponctuation, Sorbonne, 17 juin 2011. Nous avons voulu montrer que le vocabulaire de la ponctuation est partiellement le même que celui de l'accentuation : la terminologie dans les deux domaines est en grande partie à inventer.

4. Si bien qu'une recherche dans le Grand Corpus par " sec* conj* " renvoie aussi bien à l'anglais "seconde conjugation / conjugacion ", dans le corps de la grammaire (20 occurrences) qu'au latin "secunde coniugationis", abrégé en «sec. conj.» par Génin, dans les tables (470 occurrences).

5. Onoma esti meros logou ptôtikon, sôma ê pragma sêmainon [...] koinôs te kai idiôs legomenon, " Le nom est une partie de phrase casuelle désignant un corps ou une action [...] qui s'emploie avec (valeur) commune ou particulière ", trad. Lallot $1998: 51$.

6. Il est intéressant de la comparer avec la description du nom dans les grammaires latines de la même époque. On y retrouve le même principe que l'adjectif a besoin d'un support dans l'énoncé, 
avant que n'apparaisse l'idée que le substantif désigne, alors que l'adjectif qualifie, ce qui aboutira à l'éclatement de la classe nominale (cf. Colombat 1999 : 193-206).

7. « sengnfie » dans le manuscrit.

\section{RÉSUMÉS}

La grammaire française a été créée par un transfert à partir de la grammaire latine, ce qu'on peut appeler un " transfert de technologie ». Les deux sources principales d'inspiration ont été deux artes antiques : l'Ars Donati (IVe s.) et l'Ars Prisciani (VIe s.), auxquelles s'est ajouté un apport médiéval. L'objet de cet article est d'évaluer l'importance du latin et des sources latines dans la constitution des premières grammaires françaises, notamment les grammaires de FLE. Dans un premier temps, la question du latin est examinée en rapport avec une typologie des ouvrages. Le latin est important en ce qu'il reste la métalangue de beaucoup d'ouvrages de FLE, mais aussi en ce que la description de la langue française est le plus souvent rapportée, de façon différentielle, à la description traditionnelle du latin en latin. Dans un second temps, sont examinés deux cas de transfert : (1) la subdivision, dans la classe nominale, en substantif et adjectif, une subdivision qui est apportée par la grammaire médiévale et qui vient interférer avec la division initiale entre noms propres et noms communs ; (2) le traitement du pronom pour lequel il existait déjà dans la grammaire antique, chez Donat et chez Priscien, une différence dans les définitions, et donc dans la répartition des items.

French grammar was created by a transfer from Latin grammar, what one might call a "technology transfer". The two main sources of inspiration were two ancient artes: Ars Donati (4th Century) and Ars Prisciani (6th Century), supplemented by a medieval contribution. The purpose of this paper is to evaluate the importance of the Latin language and Latin sources in the creation of the first French grammars, notably grammars for FLE (Français Langue Étrangère). In the first section, the question of Latin is examined in relation to a typology of grammars. Latin is important because it is the metalanguage used in many FLE books, but also because the description of the French language is most often contrasted with the traditional description of Latin in Latin. In the second section, two cases of transfer are considered: (1) the subdivision of the nominal class into substantives and adjectives, a contribution of medieval grammar which interfered with the initial division into proper names and common nouns; (2) the treatment of the pronoun, which Donatus and Priscian defined differently, resulting in a different distribution of items.

\section{INDEX}

Keywords : adjective, français langue étrangère (FLE), French grammar, Latin grammar, metalanguage, noun, parts of speech, pronoun., Renaissance, substantive

Mots-clés : adjectif, français langue étrangère (FLE), grammaire française, grammaire latine, métalangage, nom, parties du discours, pronom., Renaissance, substantif 
AUTEUR

BERNARD COLOMBAT

UMR 7597, « Histoire des théories linguistiques », Université Paris Diderot 